\title{
Commentary: Is glue the key to success in malignant pleural mesothelioma?
}

\author{
Valerie W. Rusch, MD
}

\author{
From the Thoracic Service, Department of Surgery, Memorial Sloan Kettering Cancer Center, New York, NY. \\ Dr Rusch's work is supported in part by National Institutes of Health / National Cancer Institute Cancer Center \\ Support Grant P30 CA008748. \\ Disclosures: Author is a participant in Institutional clinical trials funded by Genentech and Genelux, Inc, serves as \\ cochair of the NIH Thoracic Malignancy Staging Committee, and is a member of the Data Safety and Moni- \\ toring Committee, MARS2 trial. \\ Received for publication Sept 10, 2019; revisions received Sept 10, 2019; accepted for publication Sept 11, 2019; \\ available ahead of print Oct 16, 2019. \\ Address for reprints: Valerie W. Rusch, MD, Memorial Sloan-Kettering Cancer Center, 1275 York Ave, New \\ York, NY 10065 (E-mail: ruschv@mskcc.org). \\ J Thorac Cardiovasc Surg 2020;159:341-2 \\ $0022-5223 / \$ 36.00$ \\ Copyright $(2019$ by The American Association for Thoracic Surgery \\ https://doi.org/10.1016/j.jtcvs.2019.09.053
}

Malignant pleural mesothelioma (MPM) remains one of the major challenges in thoracic oncology. A rare and usually fatal cancer with a tumor biology that is poorly understood, it has proved remarkably resistant to conventional therapy with surgery, chemotherapy, and radiation. Treatment options are often limited by advanced patient age and comorbidities. Because MPM usually remains confined to a single hemithorax for much of its clinical course, therapeutic efforts have focused on ways to optimize local tumor control. For patients able to tolerate surgical resection (now most frequently performed through extended pleurectomy and decortication) a bewildering array of adjunctive therapies have been studied, including preoperative or postoperative hemithoracic radiation, ${ }^{1,2}$ intrapleural chemotherapy with or without hyperther$\mathrm{mia}^{3-5}$ and intrapleural photodynamic therapy. ${ }^{6,7}$

In this issue of The Journal, Opitz and colleagues ${ }^{8}$ report the results of a phase I clinical trial of intraoperative cisplatin loaded to fibrin sprayed across resected surfaces after pleurectomy and decortication. Although the trial included only 12 patients treated across 4 escalating dose levels of cisplatin, it is based on robust preclinical data and is the result of meticulous bench to bedside research., ${ }^{9,10}$ The outcomes of this trial mirror those in earlier animal studies. The combination of surgical resection and cisplatin-fibrin was well tolerated and associated with a level of adverse events within the range expected for this operation. The trial included detailed pharmacokinetic studies. Local cisplatin concentration within chest wall tissue was high at all dose levels, while the serum concentrations were always below renal toxicity levels. Importantly, cytotoxic levels of cisplatin in chest wall soft tissue biopsy samples were sustained for days to even months after treatment. Median overall survival was 21 months, with prolonged disease-free survival in several patients, results that are among the best reported for this group of patients

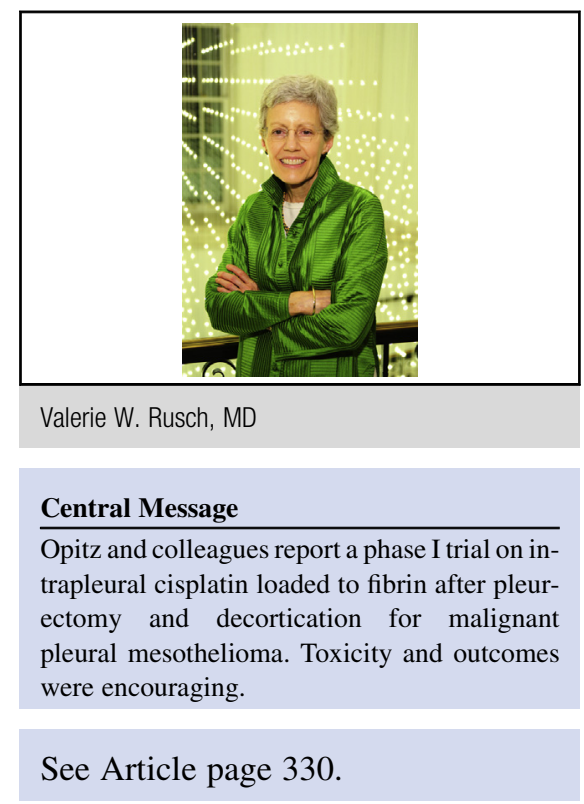

and are sufficient to justify the group's ongoing phase II trial.

Is the addition of fibrin gel to achieve protracted release of intrapleural cisplatin the key to success in achieving local control and improved outcomes in MPM? It appears to circumvent some of the toxicity seen in previous intrapleural chemotherapy trials, ${ }^{3,5}$ but many questions remain for future studies. This treatment strategy requires meticulous surgical technique and excellent team care to protect the patient and operating room staff from cisplatin-related toxicity. The lack of a direct relationship between cisplatin dose and cisplatin tissue levels raises concerns about uniformity of drug distribution and depth of tissue penetration. Although it is the chemotherapy backbone for MPM, cisplatin has modest overall activity that may not be improved in many patients by this dose-intense approach. It will be important to define not only the overall survival but also the patterns of disease progression associated with this treatment strategy. How best to integrate it with systemic therapies or radiation remains to be seen.

Opitz and colleagues ${ }^{8}$ are to be congratulated on excellent translational research leading to a novel clinical trial. Although preliminary, their results hold promise for some patients with this difficult malignancy.

\section{References}

1. Rimner A, Zauderer MG, Gomez DR, Adusumilli PS, Parhar PK, Wu AJ, et al. Phase II study of hemithoracic intensity-modulated pleural radiation therapy 
(IMPRINT) as part of lung-sparing multimodality therapy in patients with malignant pleural mesothelioma. J Clin Oncol. 2016;34:2761-8.

2. Cho BCJ, Feld R, Leighl N, Opitz I, Anraku M, Tsao M-S, et al. A feasibility study evaluating surgery for mesothelioma after radiation therapy: the "SMART" approach for resectable malignant pleural mesothelioma. J Thorac Oncol. 2014;9:397-402.

3. Rusch VW, Niedzwiecki D, Tao Y, Mendendez-Botet C, Dnistrian A, Kelsen D, et al. Intrapleural cisplatin and mitomycin for malignant mesothelioma following pleurectomy: pharmacokinetic studies. J Clin Oncol. 1992;10:1001-6.

4. Rusch VW, Saltz L, Venkatraman E, Ginsberg R, McCormack P, Burt M, et al. A phase II trial of pleurectomy/decortication followed by intrapleural and systemic chemotherapy for malignant pleural mesothelioma. J Clin Oncol. 1994;12: 1156-63.

5. Richards WG, Zellos L, Bueno R, Jaklitsch MT, Jänne PA, Chirieac LR, et al. Phase I to II study of pleurectomy/decortication and intraoperative intracavitary hyperthermic cisplatin lavage for mesothelioma. J Clin Oncol. 2006;24:1561-7.

6. Friedberg JS, Simone CB 2nd, Culligan MJ, Barsky AR, Doucette A, McNulty S, et al. Extended pleurectomy-decortication-based treatment for advanced stage epithelial mesothelioma yielding a median survival of nearly three years. Ann Thorac Surg. 2017;103:912-9.

7. Davis RW 4th, Papasavvas E, Klampatsa A, Putt M, Montaner LJ, Culligan MJ, et al. A preclinical model to investigate the role of surgically-induced inflammation in tumor responses to intraoperative photodynamic therapy. Lasers Surg Med. 2018:50:440-50.

8. Opitz I, Lauk O, Meerang M, Jetter A, Aeschlimann B, Seifert B, et al. Intracavitary cisplatin-fibrin chemotherapy after surgery for malignant pleural mesothelioma: a phase I trial. J Thorac Cardiovasc Surg. 2020;159: 330-40.e4.

9. Lardinois D, Jung FJ, Opitz I, Rentsch K, Latkoczy C, Vuong V, et al. Intrapleural topical application of cisplatin with the surgical carrier Vivostat increases the local drug concentration in an immune-competent rat model with malignant pleuromesothelioma. J Thorac Cardiovasc Surg. 2006;131:697-703.

10. Optiz I, Erne BV, Demirbas S, Jetter A, Seifert B, Stahel R, et al. Optimized intrapleural cisplatin chemotherapy with a fibrin carrier after extrapleural pneumonectomy: a preclinical study. J Thorac Cardiovasc Surg. 2011;141: 65-71. 